\title{
CURADORIA DO ACERVO PALEONTOLÓGICO DA UNIVERSIDADE FEDERAL DO RIO GRANDE
}

\author{
Débora Diniz' \\ Helena loewenstein ${ }^{2}$ \\ Paula C. Dentzien-Dias ${ }^{3}$
}

\section{RESUMO:}

O acervo do Laboratório de Geologia e Paleontologia (LGP) é formado, em sua maioria, por fósseis da Planície Costeira do Rio Grande do Sul (PCRS). Estudos vêm sendo desenvolvidos desde meados da década de 1990 sobre este acervo, a despeito dos quais muitos trabalhos ainda devem ser realizados. Para que esta coleção seja internacionalmente conhecida, um amplo trabalho de normatização tem sido desenvolvido a fim de identificar e catalogar os fósseis depositados neste laboratório. Estes fósseis foram distribuídos em 21 classes taxonômicas, cada representada por uma letra. Até o momento 5806 peças/lotes já foram devidamente identificadas e tombadas. Os táxons mais representativos são os invertebrados, principalmente Mollusca e Echinodermata. Outros táxons incluem Teleostei, Chondrichthyes, Toxodontidae, Litopterna, Gomphoteriidae, Artiodactyla, Perissodactyla, Xenarthra, Carnivora, Cetaceae, Testudinata, e Aves. $O$ grande desafio desta coleção é a normatização dos dados, visto que nunca houve uma metodologia de coleta estabelecida. Dados como procedência e data de coleta estão faltando, bem como quem os coletou e identificou. Grande parte destes fósseis foi coletada nas praias do litoral central e sul do Rio Grande do sul. Os fósseis são catalogados, etiquetados e armazenados em condições que garantam a integridade dos espécimes e sua disponibilidade para pesquisadores e estudantes. Para a continuidade do processo de identificação, parcerias dentro da instituição e com grupos da América Latina são de grande importância. Esta coleção possui um grande potencial científico, pois muitos fósseis ainda carecem de estudos aprofundados.

\section{PALAVRAS-CHAVE:}

Quaternário, Curadoria Científica, Vertebrados Marinhos e Continentais, Invertebrados marinhos, Icnofósseis

\begin{abstract}
:
The Laboratório de Geologia e Paleontologia (LGP)'s collection is mainly formed by fossils from the Rio Grande do Sul Coastal Plain (RSCP). Many studies have been developed about this collection since the 1990s, however a lot of research for new studies need to be done. For this collection to be internationally known, a wide work of normative is being developed to identify and catalog the fossils of this laboratory. These fossils were distributed in 21 taxonomic classes, each one represented by a letter. Until now, 5806 fossils/groups were identified and registered. The invertebrates are the most representative taxonomic group, specially the Phyla Mollusca and Echinodermata. Other taxa include Teleostei, Chondrichthyes, Toxodontidae, Litopterna, Gomphoteriidae, Artiodactyla, Perissodactyla, Xenarthra, Carnivora, Cetaceae, Testudinata and Birds. The biggest challenge in this collection is the normative of the data, once the laboratory has never established a collection methodology. Some data, as origin and date of collect are missing, as well as who collected and who identified. However, is known that most part of the fossils was collected on the beaches of the central and south coast of Rio Grande do Sul. The fossil specimens are catalogued, labeled, and stored in conditions that will ensure their protection and availability to researchers and students. To continue the process of identification, partnerships inside the institution and with other groups of Latin America are very important. This collection has a big scientific potential, once many fossils still need to be studied deeply.
\end{abstract}

\section{KEY-WORDS:}

Quaternary, Scientific Curation, Marine and Continental Vertebrates, Marine invertebrates, Ichnofossils.

I Doutoranda em Oceanografia Física, Química e Geológica, Instituto de Oceanografia, FURG, Rio Grande, RS. dede.p.diniz@gmail.com. Avenida Itália, s/n, Campus Carreiros, CEP 96203-900, Rio Grande, RS.

2 Graduanda em Oceanografia, Instituto de Oceanografia - FURG, Rio Grande, RS.

3 Doutora em Geociências, Instituto de Oceanografia - FURG, Rio Grande, RS. 


\section{Introdução}

Uma coleção científica deve conter os espécimes devidamente tratados, de acordo com as características do acervo; documentado, na forma de um livro tombo, para que as informações sejam preservadas; ser acessível; ter sua integridade, longevidade e qualidade asseguradas para pesquisas futuras (DOU, 2007; Carvalho, 2010). A fim de assegurar que os fósseis depositados na FURG atendessem a estes requisitos, deu-se início em 2012 a um extenso trabalho de limpeza, organização e catalogação de todos os espécimes.

As coleções paleontológicas possuem um caráter único, pois todos os espécimes são insubstituíveis, devendo ser compreendidos como registros da história da vida em nosso planeta (Carvalho, 20I0). Isto significa que todas as peças da coleção são importantes registros, não somente os holótipos, espécime tipo para a identificação de uma espécie, e parátipos, espécimes tipo pertencente ao mesmo lote do holótipo.

Além do caráter científico, estas coleções despertam grande interesse da comunidade em geral. É notável que, quando abertas ao público, as coleções paleontológicas recebem grande número de visitantes (Figurelli, 20I I;Ziemann et al., 20 I3), sejam leigos ou especialistas, além do interesse de escolas de ensino fundamental e médio (Almeida et al., 20 I3; Melo, et al., 2007; Santos e Carvalho, 20 I3).

Várias coleções paleontológicas já estão exercendo seu poder transformador e informativo sobre as comunidades residentes no entorno de universidades e museus, bem como aquelas residentes em regiões de afloramento (Kellner, 2005). Experiências assim são notáveis no Ceará (Viana et al., 2005), Piauí (Dentizien-Dias, 20I I), Espírito Santo (Almeida et al., 20I3), entre outros.

O trabalho de curadoria em uma coleção científica paleontológica não deve ser compreendido somente como responsabilidade de guarda e manutenção desta coleção (Carvalho, 2010). O curador deve também estar envolvido em atividades de ensino e pesquisa de modo a multiplicar o conhecimento dos espécimes da coleção e formar mais profissionais capacitados ao estudo paleontológico.

A preparação dos fósseis para que estes sejam englobados em coleções pode ser bem variável. Ela está diretamente relacionada com a matriz sedimentar em que o fóssil se encontra e com o objetivo da pesquisa em curso. Muitas técnicas podem ser usadas, porém não há padronização e tampouco disponibilidade de equipamentos específicos para estes procedimentos (Nobre e Carvalho, 20I0).

A coleção paleontológica de Laboratório de Geologia e Paleontologia (LGP) da Universidade Federal do Rio Grande (FURG) existe desde meados da década de 1970,porém nenhum trabalho extensivo de catalogação havia sido realizado. Somente em meados da década de 1990, houve a apresentação de monografias e projetos de iniciação científica (Buchmann \& Itusarry, 1994; Oliveira \& Itusarry, 200I; Caron et al., 2004; Lima et al., 2004; Mesquita \& Itusarry, 2004; Lopes \& Itusarry, 2006) com os fósseis desta coleção.Ao longo dos anos, com a formação de alunos de graduação dos cursos de Oceanologia e Geografia, vários métodos de catalogação foram usados, mas nenhum deles foi adotado como padrão. Este trabalho objetiva a disseminação do conhecimento adquirido e armazenado na FURG ao longo dos 40 anos de trabalhos de campo e coleta de fósseis, bem como o trabalho de curadoria que vem sendo desenvolvido nos últimos anos.

\section{Proveniência dos fósseis do LGP-FURG}

As principais áreas de coleta de fósseis estão localizadas ao longo da Planície Costeira do Rio Grande do Sul (PCRS).A formação geológica desta região está diretamente associada com as oscilações do Nível Médio do Mar (NMM) durante o Quaternário.Ao longo dos últimos 400.000 anos, os processos de regressão e transgressão marinha formaram quatro sistemas Laguna-Barreira (Villwoch \& Tomazelli, 1995) e, concomitantemente, deram margem à preservação de vestígios de animais que habitaram esta região neste intervalo de tempo.

O Sistema Laguna-Barreira I, o mais antigo, é denominada Barreira das Lombas (Figural). Embora provavelmente tenha se desenvolvido por uma região mais ampla, atual- 
mente restringe-se a uma área da porção noroeste da planície costeira. Embora no afloramento a maior parte das estruturas sedimentares primárias tenha sido destruída, é possível observar a ocorrência de estruturas biogênicas, especialmente àquelas relacionadas com o crescimento de raízes (Villwoch \& Tomazelli, 1995).

O Sistema Laguna-Barreira III (Figura I) encontra-se muito bem preservado ao longo da planície costeira e seu desenvolvimento foi fundamental para fase final de implantação do Sistema Lagunar Patos - Mirim. Esta barreira é constituída por fácies arenosas interpretadas como sendo de origem praial e marinho raso, recobertas por depósitos eólicos (Villwoch \& Tomazelli, 1995). Em muitos afloramentos é notável a ocorrência de Ophiomorpha, associados à Callichirus sp. Fósseis de mamíferos pleistocênicos também são comumente encontrados em associação com os depósitos sedimentares desta barreira (Lopes \& Buchmann, 20I I; Lopes \& Simone, 20I2; Hubbe et al., 20I3).

O Sistema Laguna-Barreira II (Figura I) corresponde ao primeiro estágio de evolução da Barreira Múltipla Complexa, responsável pelo isolamento do um gigantesco corpo lagunar, atualmente representados pela Lagoa dos Patos e Lagoa Mirim (Villwoch \& Tomazelli, 1995). Este sistema é considerado afossilffero.

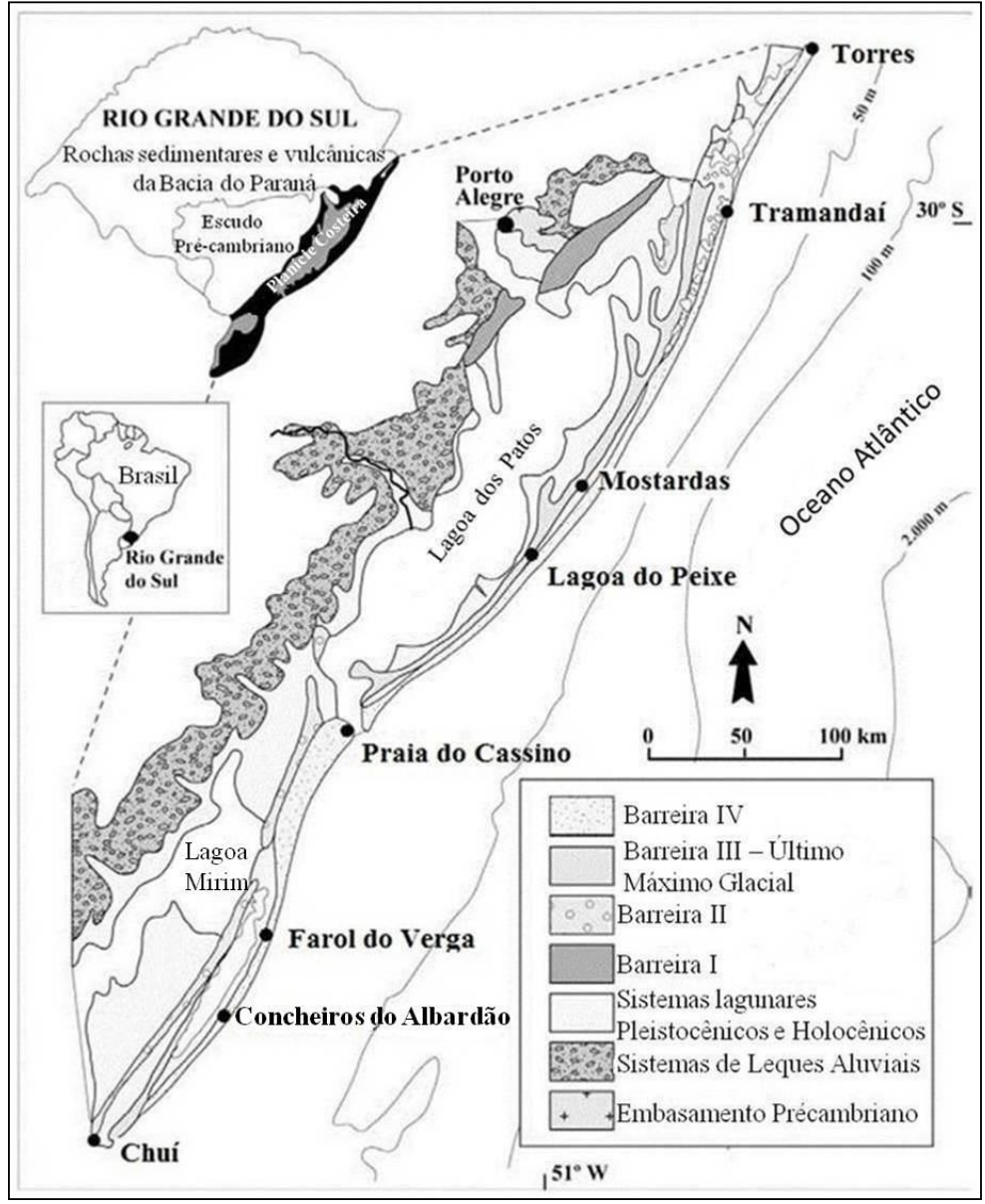

Figura I: Mapa da PCRS apresentando a extensão atual dos depostos Laguna-Barreira I a IV e os principais pontos de coleta ao longo da linha de costa. Adaptado de Rosa, Barboza et al.20I I

Por fim, a Laguna-Barreira IV desenvolveu-se durante o Holoceno (Figura I), consequência da última grande transgressão marinha pós-glacial. Durante o pico transgressivo holocênico, o NMM esteve cerca de $5 \mathrm{~m}$ acima do atual, escarpando os depósitos mais antigos e deixando depositada a sucessão de cordões litorâneos regressivos, durante a posterior regressão marinha (Villwoch \& Tomazelli, 1995).

Atualmente, vários exemplares do registro fóssil do Rio Grande do Sul podem ser encontrados depositados na superfície das praias oceânicas e lagunares do estado. Estes fósseis são oriundos de depósitos submersos, formados durante os processos de transgressão e regressão marinha, bem como por outros transportados pelos rios e lagoas 
costeiras. Estes fósseis não estão depositados em contexto geológico, sendo encontrada grande mistura de temporal. Os principais pontos onde o registro fossilifero é encontrado na PCRS estão assinalados na Figura I. Note que estão associados normalmente com os inúmeros faróis que estão instalados na linha de praia. Isso ocorre devido à falta de outros marcos topográficos ou aglomerações urbanas nesta região que possam servir de referência para o posicionamento dos pontos de coleta.

Uma pequena quantidade de fósseis provém dos depósitos mesozoicos e paleozoicos brasileiros, tendo catalogados representantes do Grupo Bauru (Cretáceo) e coprólitos da Formação Rio do Rasto (Permiano), ambos da Bacia do Paraná.

\section{Metodologia}

Quando coletados nos trabalhos de campo, os fósseis são encaminhados ao Laboratório de Geologia e Paleontologia (LGP) onde são lavados com água corrente, suficiente para remover os acúmulos de areia e secos em temperatura ambiente, quando estes são provenientes da PCRS. Os fósseis coletados nos depósitos continentais são preparados com o auxílio de cinzéis, prospectores e martelos, além de, quando necessário, é utilizado o martelo gravador. Depois de secos e limpos, um pequeno espaço nos fósseis é selecionado e pintado com tinta branca a base de água. Sobre este é escrita à sigla que o identificará junto à coleção.

As identificações seguem um padrão taxonômico, onde cada ordem, ou nível taxonômico superior, é indicado por uma letra. Cada fóssil coletado e catalogado foi tombado sob o seguinte padrão:

LGP/A - 0000,

onde:

LGP:acrônimo do Laboratório de Geologia e Paleontologia

A: letra variável que representa a identificação taxonômica da peça (Tabela I)

0000: numeração com quatro dígitos que segue a partir de $000 \mathrm{l}$ para cada nível taxonômico.

Tabela I: Grupos taxonômicos tombados no LGP.

\begin{tabular}{c|c|c|c}
\hline \multicolumn{4}{c}{ Coleção de Fósseis do LGP } \\
\hline Legenda & Taxonomia & Legenda & Taxonomia \\
LGP/A & Aves & LGP/N & Pampateriidae/Dasypodidae \\
LGP/AC & Arroio Chuí & LGP/P & Glyptodontidae \\
LGP/B & Teleostei & LGP/PC & Plataforma Continental \\
LGP/C & Chondrichthyes & LGP/Q & Mylodontidae \\
LGP/D & Outros Grupos & LGP/R & Megateriidae \\
LGP/E & Toxodontidae & LGP/S & Echinodermata \\
LGP/F & Litopterna & LGP/T & Reptilia \\
LGP/G & Proboscidae & LGP/U & Carnivora \\
LGP/H & Icnofósseis & LGP/W & Mollusca \\
LGP/I & Artiodactyla & LGP/X & Cetacea \\
LGP/K & Perissodactyla & & \\
\hline
\end{tabular}

Fonte: Laboratório de Geologia e Paleontologia da FURG.

Para melhor conservação dos fósseis foram adquiridos quatro novos armários e duas bancadas com armários inferiores, aumentando para nove os locais de armazenamento adequado de fósseis. Fósseis de tamanho mediano (até $10 \mathrm{~cm}$ ) são armazenados em caixas plásticas formando grupos com afinidade taxonômica e fósseis pequenos (como dentes e coprólitos) são armazenados em caixas plásticas e envoltos por algodão (Figura 2). 


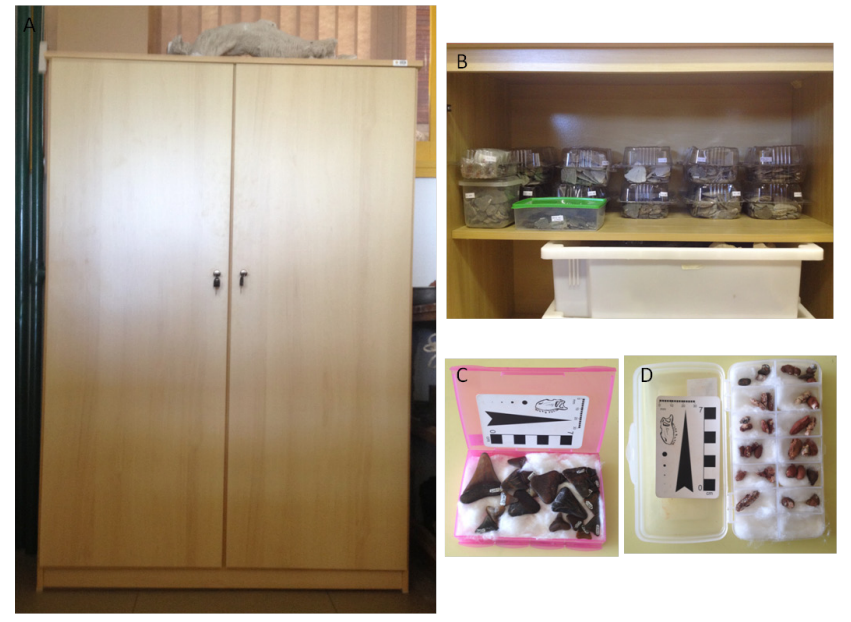

Figura 2: Armazenamento dos fóseis no LGP, após o trabalho de limpeza e identificação. Os fósseis grandes são armazenados diretamente nos armários $(A)$, os fósseis médios, em caixas plásticas (B), e aqueles de pequeno porte (menores que $10 \mathrm{~cm}$ ) em caixas organizadoras (C e D). Fonte: LGP.

Foi desenvolvido um termo de empréstimo e intercâmbio de materiais seguindo a Instrução Normativa do Ministério do Meio Ambiente (DOU, 2007). Todo termo é impresso em duas vias, sendo assinado pelo curador e pelo pesquisador. Uma via fica com o pesquisador e a outra fica armazenada junto ao Livro Tombo no LGP (Figura 3).

Além dessas normatizações da coleção, os curadores vêm realizando cursos de Curadoria e Preparação dos fósseis, a fim de otimizar o trabalho realizado.

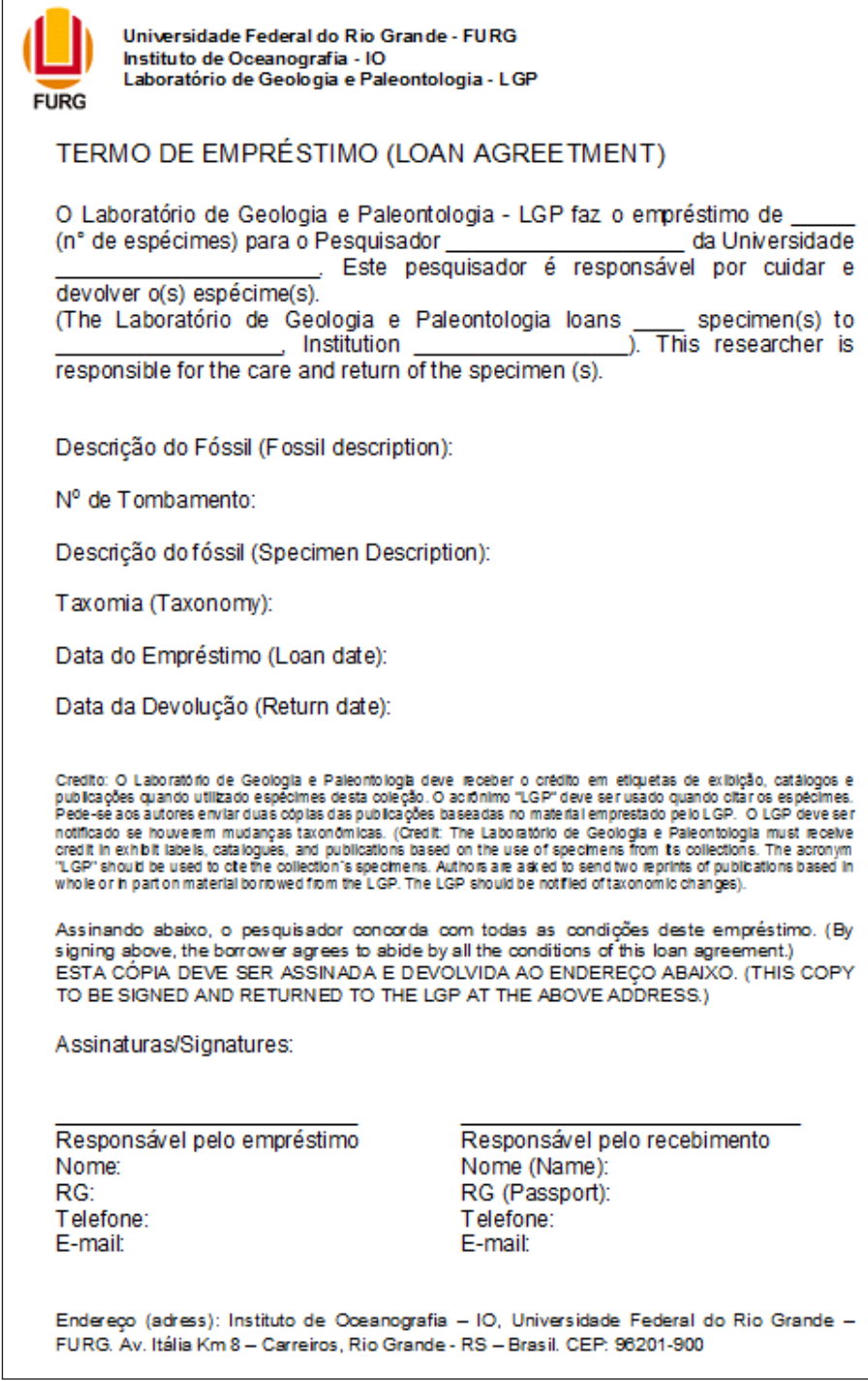

Figura 3:Termo de empréstimo usado para a sessão de material fóssil a outras universidades, museus ou pesquisadores, a fim de desenvolverem estudos referentes a estes fósseis. Note que o termo é bilíngue, facilitando a compreensão por pesquisadores estrangeiros. Fonte: LGP. 


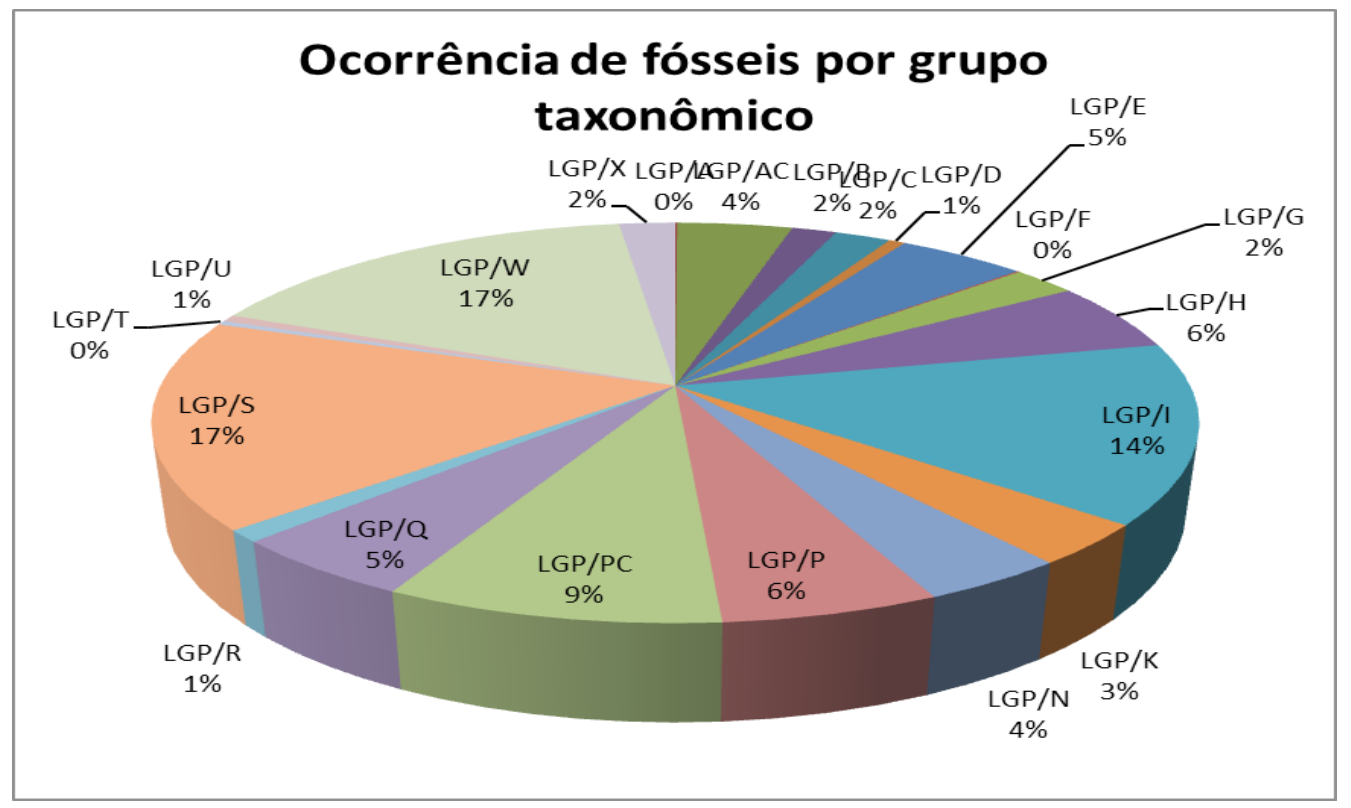

Figura 4: Representatividade de cada grupo taxonômico na coleção do LGP.

\section{Resultados}

O trabalho de identificação e tombamento dos espécimes depositados no LGP está em curso.Até o momento o LGP já possui um acervo documentado de 5806 peças, distribuídos em 21 grupos taxonômicos (Figura 4).

Dentre estes grupos taxonômicos estão representadas a megafauna sulamericana, que ocupava a região do sul do Brasil e América do Sul até cerca de 10 ka, e organismos marinhos, que tiveram seu espectro de distribuição alterado pelas variações do nível do mar e climáticas, mas que, em grande parte, ainda são encontrados na região sul do Brasil.

Mollusca (LGP/W 000I-0963) é o grupo de organismos invertebrados mais abundantes na PCRS. Os principais registros referem-se à Ostrea puelchana, O. equestris, Crassostrea rhizophorae, $C$. virginica, além de registros menos numerosos de Amiantis purpuratus, Mactra marplatensis, M. isabelleana, Zidona dufresnei,Adelomelon brasiliana, Pitar rostratus, Glycimeris longoir, entre outras. Alguns trabalhos já foram desenvolvidos no acerco malacológico do LGP sobre a predação de poríferos sobre as conchas (Lopes, 20ll), novas identificações importantes de espécies fósseis (Lopes \& Simone, 20I2) e aspectos tafonômicos dos depósitos malacológicos (Lopes \& Buchmann, 2008).

Echinodermata (LGP/S 000 I-0980) é outro filo invertebrado muito comum na plataforma continental. Grande parte dos registros refere-se equinodermos irregulares, como Encope emarginata e Melita quinquiesperforata. Estes fósseis possuem um alto nível de abrasão, indicando retrabalhamento pela dinâmica costeira.Vários processos de fossilização foram analisados e descritos (Lopes, 200 I).

Os peixes cartilaginosos, Chondrichthyes (LGP/C 000I-0I39) estão representados por dentes e placas dentárias de tubarões e raias. Até agora, encontram-se identificados dentes de Galeocerdo cuvier, Carcharodon carcharias e Myliobatiformes em geral.

Os peixes ósseos, Teleostei (LGP/B 000I-0093), compreendem, em sua maioria, registro de peixes marinhos e estuarinos. As principais espécies encontradas são Pogonias cromis e Micropogonias furnieri, além de peixes não identificados das famílias Diodontidae e Ariidae. Os principais fósseis são vértebras, 
otólitos, placas dentárias, escamas e fragmentos de opérculos.

Dentre os Reptilia (LGP/T 000I-002I) há a ocorrência somente de Testudinata (tartarugas). Pouco se estudou a respeito destas ocorrências e suas implicações paleoclimáticas para a PCRS.

Outros Grupos (LGP/D 000I-0035) é a única categoria que abrange mais um de táxon. Alguns grupos taxonômicos depositados no LGP são provenientes de doações e trabalhos de campo muito específicos, os tornando pouco abundantes. A fim de evitar um número excessivo de categorias, estes táxons foram agrupados. Os principais representantes esta categoria são Titanossauridae provenientes da Formação Marília, Membro Serra da Galga, no estado de Minas Gerais, e Braquiopoda, provenientes da Formação Ponta Grossa

Xenarthra foi subdividida em quatro categorias devido a grande quantidade de material pertencente a esta ordem. Pampateriidae e Dasypodidae (LGP/N 000 I-022I) foram tatus de grande porte que se desenvolveram na América do Sul. As principais espécies registradas para a PCRS são Pampatherium humboldti, Pampatherium typum, Holmesina paulacoutoi, Holmesina majus, Propaopus sp. e Dasypus sp.. Todos os registros correspondem a osteodermos. Glyptodontidae (LGP/P 000 I-0272) foram bastante semelhantes aos tatus gigantes e também habitaram a América do sul até a última glaciação quaternária. Os registros da PCRS englobam Glyptodon reticulatus, G. clavipes, Neuryurus rudis, Panochthus tuberculatus, Doedicurus clavicaudatus e Pachyarmatherium tenebris, em sua maioria osteodermos, além de tubos caudais e falanges. Mylodontidae (LGP/Q 000I-0292) é um grupo de preguiças gigantes extinto. Fósseis de Mylodon darwinii, Glossotherium sp., Catonix sp. e Lestodon sp. já foram coletados na PCRS. Os registros são compostos por dentes, osteodermos, astrágalos, tíbias, úmeros, dentários e metacarpais. Megateriidae (LGP/R 000I-0056) é representado somente por Megatherium sp., do qual são encontrados dentes, falanges, manúbrio, tíbia e parte de um crânio.

Os registros de Artiodactyla (LGP/I 0000I-084I) abrangem Morenelaphus sp., Antifer sp.,Ozotocrerus sp., além de vários Cervidae e Artiodactyla indeterminados.As principais ocorrências são de fragmentos de galhadas e dentes, além de astrágalos, tíbias, vértebras e naviculocubóides.

Os Notoungulata - Toxodontidae (LGP/E 000 I-0277) são mamíferos pleistocênicos exclusivos da América do Sul, que se assemelham bastante com os rinocerontes atuais (Lopes and Buchmann 20I I). Os registros deste grupo incluem principalmente dentes molares e incisivos, provenientes da região dos Concheiros do Albardão.

Os fósseis de Perissodactyla (LGP/K 000I-0194) identificados até o momento são representados, em grande maioria por dentes não identificados de Equidae. Dentre os identificados, constam dentes de Hippidion sp. e Tapirus sp.

Os Proboscidea (LGP/G 000I-0I39) são representados somente por fósseis de Stegomastodon waringi. As principais ocorrências são de molares e fragmentos de dentes, mas estão catalogados também dentários, tíbias e um processo espinhal de uma vértebra.

Cetacea (LGP/V 000I-0I20) engloba os fósseis de Mysticeti e Odontoceti. Dentre os misicetos, registram-se vértebras, fragmentos de costelas e discos intervertebrais. Para os odontocetos, os crânios de toninhas (Pontoporia blaivillei) ganham grande destaque.

Os Liptoterna (LGP/F 000 I-0005) são mamíferos extintos, endêmicos daAmérica do Sul. Os registros são compostos por dentes molares, incisivos e uma vértebra cervical de Macrauchenia patachonica, e um astrágalo de Neolicarphrium recens. 
$\mathrm{Na}$ ordem Carnivora (LGP/U 000I-0023) foram encontrados fósseis de animais marinhos e terrestres na PCRS. Para os animais marinhos há representantes das famílias Otariidae e Phocidae. Os registros terrestres, menos frequentes, são representados por dois astrágalos e um úmero, identificados como sendo Smilodon populator.

$\mathrm{Na}$ classe Aves (LGP/A - 000I-0004) existem somente quatro espécimes catalogados, identificados como três vértebras cervicais e um tibiotarso esquerdo (Lopes et al., 2006), pertencentes à família Thalassarche.

Os Icnofósseis (LGP/H 000I-03 I0) pertencentes à coleção, em sua maioria, são traços fósseis de organismos que ocupam a região costeira. São encontrados rolados nas praias, transportados de regiões mais profundas pelas ondas e correntes costeiras. Cerca de $90 \%$ destes registros correspondem a Ophiomorpha, tocas horizontais e verticais características de ambientes rasos, que possuem uma estrutura externa formada por pellets, agrupados de várias formas (Frey et al., 1978; Pollard et al., 1993). Coprólitos provenientes da Formação Rio do Rasto (Dentzien-Dias et al., 20II), da Bacia do Paraná também enriquecem a coleção.

Os fósseis procedentes do Arroio Chuí (LGP/AC 000I-0247) foram coletados ao longo de várias campanhas de campo. Esta região é considerada um dos principais afloramentos fossilíferos costeiros do Brasil (Lopes et al., 2009).As peças desta categoria não foram identificadas taxonomicamente até o momento. Isto ocorre devido a grande fragmentação destas peças, a similaridade de alguns fósseis de grupos distintos e da carência de especialistas neste laboratório para identificar os fósseis. A fim de minimizar estas questões, parcerias estão sendo estabelecidas com pesquisadores de diversas instituições para que o processo de identificação seja o mais preciso possível.

Todos os fósseis que não possuem identificação taxonomia, exceto os provenientes do Arroio Chuí, estão incluídos na divisão 'Plataforma Continental' (LGP/PC 000I - 0529). Estes fósseis são provenientes de todos os pontos de coleta ao longo da PCRS.

Além de todas estas peças identificadas, estão depositadas no LGP algumas caixas contento centenas de fósseis que ainda carecem de identificação. Dentre estes fósseis estão peças de grande valor, seja pelo estado de preservação, seja pela relevância delas para o registro das modificações morfológicas da PCRS e das mudanças climáticas ocorridas ao longo do Quaternário. Dessa forma, toda semana novos fósseis e icnofósseis estão sendo identificados e adicionados à coleção do LGP.

O desenvolvimento do termo de empréstimo do LGP foi fundamental para a organização de identificação de todas as peças que estão temporariamente sob guarda de outras instituições e pesquisadores. Além disso, contém todos os dados de contato e tempo pelo qual as peças ficaram emprestadas.

\section{Discussão}

A partir da identificação das demandas existentes no LGP, como a normatização, a disponibilização de espaço e o processo de identificação das peças, algumas medidas importantes têm sido adotadas.

Os procedimentos de coleta foram estabelecidos de forma a contemplar todas as necessidades. São anotados dados como data (possibilidade de associação com condições climáticas), coletor e local (para associação com condições de transporte e deposição). É necessário ressaltar que, apesar de serem informações simples, a falta destas compromete os trabalhos científicos que se basearão nestes fósseis. 
O processo de identificação dos fósseis está sendo realizado em parceria com outros pesquisadores da FURG e de outras instituições. A FURG possui grande número de especialistas em Oceanografia Biológica e Biologia, com grande conhecimento dos táxons e de sua distribuição atual. Para os grupos da megafauna pampeana, extintos no fim do Pleistoceno, contatos com instituições e grupos latino-americanos, que possuem experiência internacionalmente reconhecida estão sendo estabelecidas. Uma visita à coleção do Museo de La Plata, vinculado a Universidad Nacional de La Plata já foi realizada e outras estão previstas.

Apesar de todos os cuidados com os procedimentos de coleta, as amostras ainda devem ser analisadas quanto à origem geológica. A plataforma continental do Rio Grande do Sul possui alguns paleoníveis de praia (Corrêa, 1996), que estão relacionados a estabilizações no NMM durante a última transgressão marinha pleistocênica. Estes paleoníveis estão, atualmente, sendo retrabalhados pela dinâmica de ondas e correntes da plataforma, fazendo com que seu conteúdo fossilífero seja redisponibilizado e transportado até alcançar a atual linha de praia. Quanto se toma nota da posição de coleta do fóssil não é possível determinar com precisão por quanto tempo ele foi transportado e de qual destas paleopraias é proveniente. Algumas inferências baseadas em análises tafonômicas e no estado de preservação do fóssil podem ser feitas, mas a determinação mais aproximada de idade fica comprometida. As características tafonômicas são um importante meio de buscar identificar o ambiente no qual o organismo foi fossilizado. Analisando características como coloração, substituição química, trincas, e outras marcas, é possível associar o fóssil a um ambiente e, a partir deste, estabelecer uma idade relativa (Simões et al., 2010).

\section{Conclusão}

A curadoria de uma coleção científica, independente de sua natureza, envolve muito mais que somente a limpeza e manutenção do material. Cuidados na coleta, identificação, pesquisa científica e ensino também estão diretamente relacionados com as atividades de curadoria.

A coleção de fósseis do Laboratório de Geologia e Paleontologia da FURG é muito rica, contemplando, principalmente, grupos taxonômicos que habitaram o Quaternário do sul do Brasil. Grande parte deste acervo ainda carece de identificação, estando tombando em classes generalistas como Arroio Chuí e Plataforma Continental.

Além dos grupos quaternários, estão depositados no LGP fósseis provenientes de outros períodos geológicos. Entretanto, somente estão tombados fósseis de dinossauros do Cretáceo e coprólitos do Permiano. Todavia encontram-se no LGP exemplares de vertebrados proveniente do Triássico e Permiano gaúcho, além de fósseis doados por instituições estrangeiras, coletados a mais de cinco anos, ainda sem número de tombamento. Desta forma fica claro que a organização do laboratório é de suma importância, visto que até o momento não há catalogação destes exemplares.

O potencial do laboratório como gerador de pesquisas científicas é evidenciada quando observada a vastidão do acervo. Com as parcerias internas, com pesquisadores da própria universidade, e parcerias externas, como o Museo de La Plata na Argentina, a produção científica tende a ampliar-se, apresentado importantes resultados para o estudo das mudanças climáticas durante o Quaternário.

A partir de 2012 a curadoria da Coleção Paleontológica do LGP- FURG está sendo realizada com o maior cuidado para que o potencial desta coleção seja reconhecido nacional e internacionalmente como uma das mais completas acerca do Quaternário Marinho e Terrestre da América do Sul. O desenvolvimento de um Termo de Empréstimo do LGP foi fundamental para a organização da coleção e para evitar a perda de materiais. $O$ armazenamento correto da coleção busca mitigar os 
impactos nocivos de um mau salvamento dos fósseis, evitando quebras e perdas dos fósseis, e facilitando o trabalho dos curadores e pesquisadores.

\section{Agradecimentos}

Os autores gostariam de agradecer ao Dr. Renato Lopes, Dr. Francisco Buchmann e Dr. Felipe Caron, que trabalham com o acervo desta coleção e se disponibilizaram a auxiliar na busca por importantes informações. A MSc. Ana Emília de Figueiredo, Dr. Leonardo Ávila, MSc. Dimila Mothé, MSc. Mariela Castro, Biol. Mara Loza e MSc.Victor Eduardo Pauliv pelo auxílio na identificação do material.A Fapergs, através do edital ARD 03/20I2, Processo 12/2559-7.Ao CNPq, através do proceso PIBIC/FURG: I467I8/20I3-5, vigente até 3I/7//4.

\section{Referências Bibliográficas}

ALMEIDA, L. F.;ZUCON, M. H.;SOUZA,J. F.; REIS,V.S.;VIEIRA, F.S. Ensino de Paleontologia: uma abordagem não-formal no Laboratório de Paleontologia da Universidade Federal de Sergipe. TERRA DIDATICA, Campinas, v. I0, p. I4-2I, 2013.

BUCHMANN, F. S. C.; ITUSARRY, M. E. G. S. Estudo macrofossilifero na porção sul da planície costeira do Rio Grande do Sul, Brasil. 1994. 82 f. Monografia de Graduação (Oceanografia) - Universidade Federal do Rio Grande. Rio Grande, RS. 1994.

CARON, F.; ITUSARRY, M. E. G. S. Aspectos taxonômicos e tafonômicos dos fósseis de vertebrados terrestres (megafauna pampeana extinta) e marinhos (peixes e cetáceos) da Praia do Cassino, RS. 2004. I 29 f. Monografia de Graduação (Oceanografia) - Universidade Federal do Rio Grande. Rio Grande, RS. 2004

CARVALHO, I. S. Curadoria Paleontológica. In: Carvalho I. S. (Org.). Palentologia. Rio de Janeiro: Interciência, 2010. p. 397-394

CORRÊA, I. C.S. Les variations du niveau de la mer durant les derniers 17.500 ans BP: l'exemple de la plate-forme continentale du Rio Grande do Sul-Bésil. Marine Geology, v. I30, n. I, p. I63-I78. 1996.

DENTZIEN-DIAS, P. C., PRALOM, B. G. N.; SOARES,A.V. S.; FIGUEIREDO,A. E. Q.; LEAL, M. S. Coleção didática de icnofósseis da UFPI-CSHNB, Picos, Piauí. In: CarvaIho, I. S. C.; Srivastava, N. K.; Lana, C. C. Paleontologia: Cenários da Vida. Rio de Janeiro: Interciência, p. 733-740. $201 \mathrm{I}$.

DOU (2007). Instrução Normativa 160. Ministério do Meio Ambiente, Instituto Brasileiro do Meio ambiente e Recursos Renováveis. Diário Oficial da União, v. 82, n. I, p. 404-405. 2007.

FIGURELLI, Gabriela R.Articulações entre educação e museologia e suas contribuições para o desenvolvimento do ser humano. Revista Museologia e Patrimônio, v. 4, n. 2, p. 97-109, 2011.

FREY, R.W.; Howard, J. D.; Pryor,W. A. Ophiomorpha: its morphologic, taxonomic, and environmental significance. Palaeogeography, Palaeoclimatology, Palaeoecology, v. 23, n. I, p. 199-229. 1978.

HUBBE, A.; Hubbe, M.; Neves, W. A. The brazilian megamastofauna of the Pleistocene/ Holocene transition and its relationship with the early human settlement of the continent. Earth-Science Reviews,v. I I8, n. I, p. I-I0 I I 8(I): I-I0.20 I3.

KELLNER,A.W.A. Museus e a divulgação científica no campo da paleontologia.Anuario do Instituto de Geociencias, Rio de Janeiro, v. 28, n. I, p. I I 6-I30. 2005.

LIMA, L. G.; Itusarry, M. E. G. S. Perfil topo-batimétrico da Lagoa Mangueira e adjacências e aspectos estratigráficos relacionados. 2004. 1 I 5 f. Monografia de Graduação (Oceanologia) - Universidade Federal do Rio Grande, Rio Grande, RS. 2004.

Lopes, R. P. Fossil sand dollars (Echinoidea: Clypeasteroida) from the southern brasilian coast. Revista Brasileira de Paleontologia, v. I4, n. 2, p. 20I-2I4. 200 I.

Lopes, R. P. Ichnology of fossil oysters (bivalvia, ostreidae) from the southern Brazil- 
ian coast. Gaea - Journal of Geoscience, v. 7, n. 2, p. 94-I03. 20 I I.

Lopes, R. P.; Buchmann, F. S. Comparação tafonômica entre duas concentrações fossilíferas (shell beds) da Planície Costeira do Rio Grande do Sul, Brasil. GAEA - Journal of Geoscience, v.2, p. 65-77. 2008.

Lopes, R. P.; Buchmann F. S. Pleistocene mammals from the southern Brazilian continental shelf.Journal of South American Earth Sciences, v. 3I, n. I, p. I7-27. 20 I I.

Lopes, R. P.; Buchmann F. S.; Caron, F.; Itussary, M. E. Barrancas fossilíferas do Arroio Chuí, RS: Importante megafauna pleistocênica no extremo sul do Brasil. In:Winge, M.; Schobbenhaus, C.; Berbert-Born, M.; Queiroz, E.T.; Campos,D.A.; Souza,C. R. G.; Fernandes,A. C. (Org.). SSIGEP - Sítios Geológicos e Paleontológicos do Brasil. Brasília: CPRM, 2009, p. I-I0.

LOPES, R. P.; BUCHMANN, F.S. C., CARON, F. Primeiro registro de fósseis de aves marinhas na planície costeira do Rio Grande do Sul, Brasil. Revista Brasileira de Geociências, v. 36, n. 4, p. 648-650. 2006.

LOPES, R. P.; ITUSARRY, M. E. G. S. Os depósitos fossiliferos na porção sul da planície costeira do Rio Grande do Sul : aspectos paleontológicos e estratégicos. 2006. 82f. Monografia de Graduação (Geografia) - Universidade Federal do Rio Grande,Rio Gande, RS. 2006

LOPES, R. P.; SIMONE, L. R. L. New fossil records of Pleistocene marine mollusks in southern Brazil. Revista Brasileira de Paleontologia, v. I5, n. I, p.49-56. 2012.

MELO, D. J.; BASTOS, A. N. F.; RODRIGUES, V. M. C.; MONÇÃO,V. M. Desenvolvimento de atividade lúdica para o auxílio do ensino e divulgação científica da Paleontologia. Anuário do Instituto de Geociências, Rio de Janeiro, v. 30, n. I, p. 73-76. 2007. MESQUITA, H. R. O.; ITUSARRY, M. E. G. S. Evolução holocênica da paleoembocadura da Laguna dos Patos.2004, 80 f. Monografia de Graduação (Oceanografia) - Universidade Federal do Rio Grande, Rio Grande, RS. 2004.

NOBRE, P. H.; CARVALHO, I. S. Fósseis: coleta e métodos de estudo. In: Carvalho, I. S. Paleontologia. Rio de Janeiro: Insterciência, 20 I0, p. 397-4I I.

OLIVEIRA, U. R.; ITUSARRY. M. E. G. S. Índices de similaridade aplicados a fósseis de mamíferos terrestres do Pleistoceno coletados na atual linha de Costa de Santa Vitoria do Palmar (RS). 200I. 43 f. Monografia de Graduação (Oceanografia) - Universidade Federal do Rio grande, Rio Grande, RS. 200I.

POLLARD,J. E.; GOLDRING, R.; BUCK, S. G. Ichnofabrics containing Ophiomorpha: significance in shallow-water facies interpretation. Journal of the Geological Society, London. Journal of the Geological Society, London, v. I50, n. I, p. I49-I64. 1993.

SANTS, W. F. S. S; CARVALHO, I. S. Percepção dos professores do entorno do Parque Paleontológico de São José de Itaboraí (RJ) sobre aspectos geológicos, paleontológicos e arqueológicos locais TERR/E DIDATICA, Campinas, v. 9, n. I, p. 50-62. 2013. SIMÕES, M. G.; RODRIGUES S. C. Tafonomia: processos e ambientes de fossilização. In: Carvalho, I. S. Paleontologia: conceitos e métodos. Rio de Janeiro: Interciência, p. I947. 2010.

VIANA, M. S. S.; GIRÃO, G. G. S. M.; SILVA, S.W.V.; ROCHA, A R. M. O acervo fossilífero do museu Dom José (Sobral-CE) e sua importância para a divulgação da paleontologia no estado do Ceará. Revista de Geologia, v. I8, n. I, p. 53-59, 2005.

VILLWOCH, J.A.;TOMAZELLI L. J. Geologia costeira do Rio Grande do Sul. Notas Técnicas, Porto Alegre, v. 8, n. I, p. I-45. 1995.

ZIEMANN, D. R.; MÜLLER, R.T.; PACHECO, C. P.; RANGEL,A. O.; SILVA, S. D.A paleontologia como estímulo à visitação museológica: uma experiência no pampagaúcho. Revista Museologia e Patrimônio, v. 6, n. I, p. I5I-I60.20I3. 